\title{
Complete response associated with lenalidomide and celecoxib in a case of primary refractory Hodgkin lymphoma
}

This article was published in the following Dove Press journal:

OncoTargets and Therapy

\author{
Marta Garcia-Recio ${ }^{1,2}$ \\ Jordi Martinez-Serra' \\ Francesc Mestre ${ }^{2,3}$ \\ Leyre Bento ${ }^{1,2}$ \\ Jordi Gines ${ }^{4}$ \\ Rafael Ramos ${ }^{2,5}$ \\ Jaime Daumal ${ }^{2,6}$ \\ Paloma López ${ }^{2,3}$ \\ Antonia Sampol' \\ Antonio Gutierrez ${ }^{1,2}$ \\ 'Hematology Department, ${ }^{2}$ Lymphoma \\ Unit, ${ }^{3}$ Radiotherapy Department, \\ ${ }^{4}$ Pharmacy Department, ${ }^{5}$ Pathology \\ Department, ${ }^{6}$ Nuclear Medicine \\ Department, Son Espases University \\ Hospital, IdISBa, Palma, Spain
}

Correspondence: Antonio Gutierrez Hematology Department, Son Espases University Hospital, Ctra de Valldemossa, 79, Palma de Mallorca 07010, Spain $\mathrm{Tel}+3487$ I 205000 ext 65II5

Email antoniom.gutierrez@ssib.es

\begin{abstract}
Hodgkin lymphoma (HL) represents $\sim 11 \%$ of all lymphoma cases. This disease occurs in young adults, but also affects people over 55 years of age. Despite the fact that $>80 \%$ of all newly diagnosed patients under 60 will achieve a sustained complete response (CR), $5 \%-10 \%$ of HL patients are refractory to initial treatment and $10 \%-30 \%$ of patients will eventually relapse after an initial CR. The treatment recommendation for primary refractory or relapsed HL patients is salvage therapy followed by high-dose chemotherapy and autologous stem cell transplantation. Following this approach, a significant part will still relapse at any moment. Thus, further research and new drugs or combinations are required. Overexpression of COX-2 has been associated with poor prognosis in relapse/refractory HL patients, so it could be a potential therapeutic target in HL. For this purpose, several drugs may have a role: specific COX-2 inhibitors such as celecoxib or other anti-inflammatory drugs such as lenalidomide may further inhibit lipopolysaccharide-mediated induction of COX-2. Moreover, lenalidomide and COX-2 inhibitors (celecoxib) have been tested in solid tumors with encouraging results. We present a case of a young female diagnosed with a heavily pretreated HL nodular sclerosis subtype who, after failing six treatment lines, only achieved clinical and radiological CR after six cycles of lenalidomide/celecoxib that resulted in an event-free survival of 22 months. We explain the rationale of using this chemotherapy regimen and our patient follow-up.
\end{abstract}

Keywords: Hodgkin lymphoma, relapse, celecoxib, lenalidomide, COX-2

\section{Introduction}

Hodgkin lymphoma (HL) represents $\sim 11 \%$ of all lymphomas in the US. ${ }^{1}$ This disease presents with a bimodal distribution (young adults and $\geq 55$-year-old patients). Thanks to advances in HL treatment, $>80 \%$ of all newly diagnosed patients under 60 years will be cured of their disease. Despite these optimistic results, $5 \%-10 \%$ of HL patients show refractoriness to initial treatment and $10 \%-30 \%$ of patients will eventually relapse after achieving an initial complete response (CR). ${ }^{2}$

In primary refractory disease, conventional salvage therapy followed by high-dose chemotherapy and autologous stem cell transplantation is the standard of care in fit patients. Nevertheless, the 5-year freedom from failure and overall survival are $31 \%$ and $43 \%$, respectively, for these patients, which means that the vast majority will relapse even when intensive chemotherapy is used., ${ }^{2,3}$ At this point, new drugs such as brentuximab vedotin, anti-PD1, PI3K or mTOR inhibitors have shown their role in monotherapy or as a bridge to allogeneic stem cell transplantation in this subgroup of patients. ${ }^{4-6}$ However, there are no clear treatment recommendations for patients who are not eligible for intensive regimens or allogeneic stem cell transplantation. 
We present a 35-year-old woman with a diagnosis of primary refractory HL nodular sclerosis (NS) subtype, with a continuous pattern of relapses or partial responses and not considered a candidate for intensive chemotherapy or SCT. After several regimens (including radiotherapy), our patient achieved a CR to a combination regimen of lenalidomide and celecoxib. We discuss the rationale behind the use of these new drugs in relapsed HL.

\section{Case presentation}

A 35-year-old mentally disabled woman with a psychiatric history of obsessive behavior in childhood and anxiety disorder consulted the dermatology department for generalized pruritus. She was diagnosed with atopic dermatitis which resolved after corticoid-based therapy. In 2005, she was referred to our department because of laterocervical lymphadenopathies and night fever with intense sweating. Body computed tomography (CT) showed supra- and infradiaphragmatic lymphadenopathies with various abdominal masses, the most relevant being $8 \times 6 \times 8 \mathrm{~cm}^{3}$. A biopsy of a lymphadenopathy revealed NS classic HL. With a final diagnosis of NS HL stage IIIB with an International Prognostic Score 2, she received six cycles of ABVD chemotherapy regimen with partial response and an early relapse in March 2006 (primary refractory to ABVD). This was followed by a salvage chemotherapy regimen (two cycles of ESHAP) with no response. In September 2006, a third chemotherapy regimen (four cycles of GemOx) was initiated with neutropenia and thrombocytopenia grade 4, which led to doseintensity failures followed by chemotherapy discontinuation and radiotherapy consolidation. One month after the last radiotherapy session, the HL progressed; so, two cycles of IFE regimen were given with stable disease. Considering the basal comorbidities of the patient, we decided to pursue a watch-and-wait policy from 2008 to 2010, treating localized areas of progression with radiotherapy and obtaining transient partial responses (Figure 1). In October 2010, a new mass appeared in D8-D9, presenting with a medullar compression syndrome. After a biopsy that confirmed HL relapse, it was treated with radiotherapy and six cycles of GemOx, which led to a partial response. However, a new progression occurred in the left cervical and axillary nodes within 1 month, which was observed with a watch-and-wait policy with the consent of the family.

In May 2012, our patient presented a new clinically symptomatic disease progression confirmed by positron emission tomography (PET)/CT, requiring bendamustine $90 \mathrm{mg} / \mathrm{m}^{2}$ and finally reaching disease stability. In August 2014,

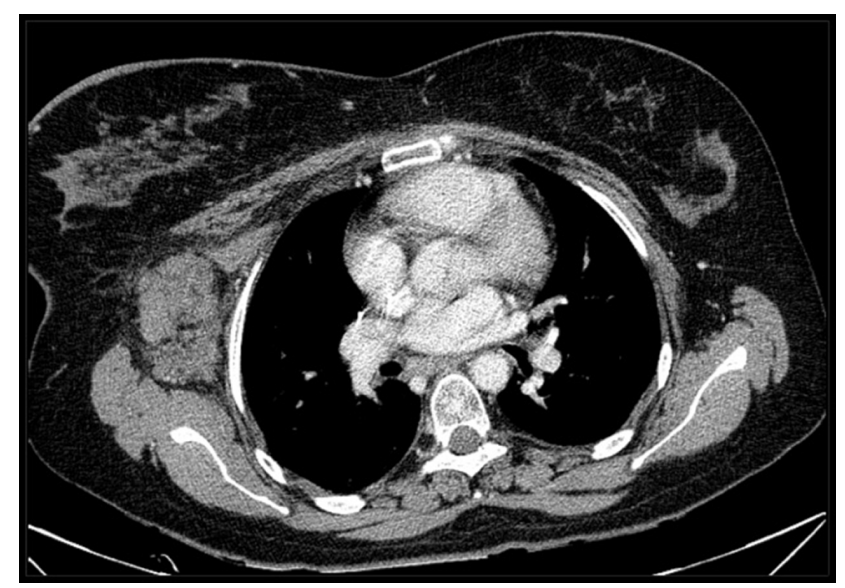

Figure I CT scan showing an axillary node transiently controlled with radiotherapy in June 2009.

Abbreviation: CT, computed tomography.

the HL progressed again with significant B symptoms and worsening clinical status (Figure 2A). At this point, based on our experience of the potential role of COX-2 expression in $\mathrm{HL}^{7,8}$ and that the patient was not a candidate for intensive chemotherapy and brentuximab was still not available in our center, we decided to start an experimental treatment with celecoxib $200 \mathrm{mg}$ every 12 hours and lenalidomide $20 \mathrm{mg}$ (for 3 of every 4 weeks). This regimen was obtained through a compassionate use request. Our patient received this treatment from August 2014 to January 2015 (six cycles) with unremarkable toxicity and excellent tolerance, finally showing a CR for the first time in both the interim after three cycles and the final CT/PET after six cycles (Figure 2B). This response was maintained in a new CT scan in August 2015. After this induction, we started 1 year of celecoxib maintenance ( $200 \mathrm{mg} / 12$ hours) that had to be stopped because of anemia due to gastrointestinal bleeding in March 2016.

In July 2016, with the most durable response until that moment being 22 months, our patient presented a new disease relapse. CT/PET showed supra- and infra-abdominal adenopathies with associated hepatic and splenic lesions. As two different biopsies of hypermetabolic locations resulted negative and keeping in mind the asymptomatic situation of our patient, we decided to restart celecoxib $200 \mathrm{mg}$ every 12 hours. In this context, the disease progressed to ascites requiring paracentesis. Biopsies were constantly negative until February 2017, when a final biopsy confirmed the persistence of HL. At that point, we decided to start brentuximab vedotin (two cycles). During the second cycle of brentuximab, the patient required intensive care admission due to a sepsis with a fatal outcome. 

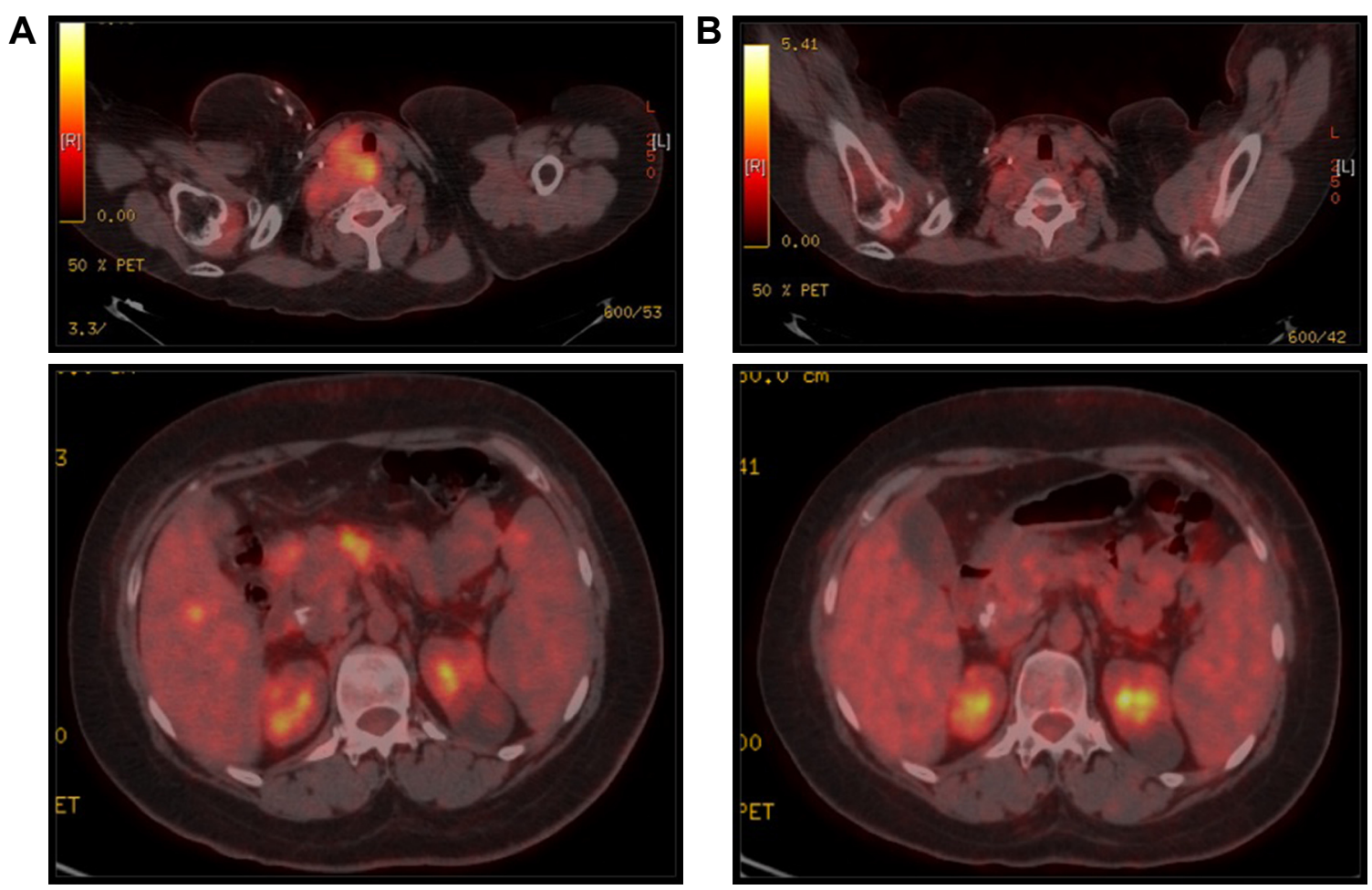

Figure 2 PET/CT scan before lenalidomide/celecoxib (A) and after six cycles of lenalidomide/celecoxib (B).

Abbreviations: $\mathrm{CT}$, computed tomography; PET, positron emission tomography.

\section{Discussion}

Patients with HL refractory to standard chemotherapy regimens represent a challenge, and even more do those patients who cannot receive intensive chemotherapy regimens or autologous stem cell transplantation. Thus, the management of these patients requires both active and well-tolerated drugs. We present a case in which lenalidomide combined with celecoxib provided a sustained $\mathrm{CR}$ in a patient refractory to radiotherapy and six prior chemotherapy lines. After six cycles of lenalidomide $20 \mathrm{mg}$ for 3 of every 4 weeks and celecoxib $200 \mathrm{mg} / 12$ hours, followed by celecoxib maintenance, our patient achieved a CR confirmed by PET/TC that lasted almost 2 years.

Our rationale for using a COX-2 inhibitor (celecoxib) is based on a study published by Mestre et al which concluded that the expression of COX-2 on Reed-Sternberg cells is an independent unfavorable prognostic factor in HL patients. ${ }^{7}$ This study suggested that COX-2 could be a new potential therapeutic target in these patients with poor prognosis. ${ }^{9}$ Other authors such as Barisik et al demonstrated a significant relationship between COX-2 expression and mixed cellular and NS HL subtypes with worse prognosis. ${ }^{10}$ COX-2 inhibitors have already been tested in solid tumors and have been found to significantly increase the overall response rate. ${ }^{11}$
Celecoxib generates a direct COX-2 inhibition that stops the conversion of arachidonic acid to prostaglandin as well as downregulates antiapoptotic genes, induces proapoptotic molecules and potentiates the formation of the apoptosome and processing of caspase-9 (cox-2-independent mechanism). Recently, celecoxib has been proposed as a good partner of CD19 CAR T-cell therapy in non-Hodgkin lymphomas due to its regulatory effect on apoptosis. ${ }^{12}$

On the other hand, lenalidomide not only acts through immunomodulatory, antiangiogenic and antiproliferative mechanisms, but also is an anti-inflammatory drug that inhibits lipopolysaccharide-mediated induction of COX-2. ${ }^{13}$ Due to these properties of lenalidomide and considering how increased neoangiogenesis and impaired immunity critically contribute to HL, Böll et al tested lenalidomide as a single agent in a continuous dosing schedule in $12 \mathrm{HL}$ patients who had relapsed after at least four chemotherapy regimens. They evidenced benefit from the use of lenalidomide in all patients. Despite none showing radiological evidence of progression after two cycles, two of the four responding patients had evidence of progression after 2 or 4 months. Overall response rate (ORR) observed was $50 \%$, including one patient with complete remission. ${ }^{14}$ Other series and clinical cases have been reported with variable ORR (19\%-50\%) and a median duration of response of 6 months even with a continuous monthly 
administration. ${ }^{15,16}$ Thus, we consider that just six cycles of lenalidomide may not fully justify the long-term remission reported here, giving special relevance to the celecoxib combination and maintenance. Moreover, the disease relapse occurred soon after celecoxib discontinuation.

Considering all these data and the evidence of good results obtained with the lenalidomide/celecoxib combination in several malignancies (prostate cancer, ${ }^{17}$ sarcomas $^{18}$ and hematological malignancies such as multiple myeloma ${ }^{19}$ or blastic plasmacytoid dendritic cell neoplasm), ${ }^{20}$ we decided to initiate this regimen. After three and six cycles of lenalidomide/celecoxib, we obtained a clinical, metabolic and radiological $\mathrm{CR}$, which was the only and longest obtained one in this patient, and the subsequent relapse occurred 4 months after discontinuing celecoxib. Considering all potential mechanisms of celecoxib-mediated apoptosis ${ }^{12}$ in the context of a patient with HL with a continuous pattern of relapses, we hypothesize that celecoxib maintenance had a vital role in controlling the disease after the induction therapy.

\section{Conclusion}

HL is a disease with poor prognosis when relapse or progression occurs. Despite advances in salvage therapy, patients who are not candidates for intensive chemotherapy, or cannot reach/maintain $\mathrm{CR}$, still have a very poor survival with no specific treatment.

We present preliminary evidence of activity of the combination of lenalidomide and celecoxib in a heavily pretreated HL patient who was not a candidate for intensive chemotherapy approaches. This combination was well tolerated and without significant toxicity. Our patient reached a 22-month CR. However, the precise role of this combination, as well as of celecoxib and lenalidomide in this case, should be confirmed in clinical trials.

\section{Consent statement}

Written informed consent has been obtained from the patient's relative (father) to publish all the case details and images needed.

\section{Acknowledgment}

We thank Mr Jonathan McFarland for his contribution to English language revision of the manuscript.

\section{Disclosure}

The authors report no conflicts of interest in this work.

\section{References}

1. Ansell SM. Hodgkin lymphoma: diagnosis and treatment. Mayo Clin Proc. 2015;90(11):1574-1583.

2. Ansell SM, Lymphoma H. Hodgkin lymphoma: 2016 update on diagnosis, risk-stratification, and management. Am J Hematol. 2016; 91(4):434-442.

3. André M, Henry-Amar M, Pico JL, et al. Comparison of high-dose therapy and autologous stem-cell transplantation with conventional therapy for Hodgkin's disease induction failure: a case-control study. Société Francaise de Greffe de Moelle. J Clin Oncol. 1999;17(1): 222-229.

4. Armand P, Shipp MA, Ribrag V, et al. Programmed death-1 blockade with pembrolizumab in patients with classical Hodgkin lymphoma after brentuximab vedotin failure. J Clin Oncol. 2016;34(31):3733-3739.

5. Younes A, Gopal AK, Smith SE, et al. Results of a pivotal Phase II study of brentuximab vedotin for patients with relapsed or refractory Hodgkin's lymphoma. J Clin Oncol. 2012;30(18):2183-2189.

6. Johnston PB, Inwards DJ, Colgan JP, et al. A Phase II trial of the oral mTOR inhibitor everolimus in relapsed Hodgkin lymphoma. Am J Hematol. 2010;85(5):320-324.

7. Mestre F, Gutierrez A, Ramos R, et al. Expression of COX-2 on Reed-Sternberg cells is an independent unfavorable prognostic factor in Hodgkin lymphoma treated with ABVD. Blood. 2012;119(25): 6072-6079.

8. Mestre F, Gutiérrez A, Rodriguez J, et al. Radiation therapy overcomes adverse prognostic role of cyclooxygenase-2 expression on Reed-Sternberg cells in early Hodgkin lymphoma. Int J Radiat Oncol Biol Phys. 2015;92(1):84-90.

9. Koh YW, Park C, Yoon DH, Suh C, Huh J. Prognostic significance of COX-2 expression and correlation with Bcl-2 and VEGF expression. Am J Surg Pathol. 2013;37(8):1242-1251.

10. Barisik NO, Bozkurt S, Gumus M, et al. Expression and prognostic significance of Cox-2 and p-53 in Hodgkin lymphomas: a retrospective study. Diagn Pathol. 2010;5:19.

11. Chen J, Shen P, Zhang XC, Zhao MD, Zhang XG, Yang L. Efficacy and safety profile of celecoxib for treating advanced cancers: a meta-analysis of 11 randomized clinical trials. Clin Ther. 2014;36(8):1253-1263.

12. Dinh TN, Onea AS, Jazirehi AR. Combination of celecoxib (Celebrex $\left.{ }^{\circledR}\right)$ and CD19 CAR-redirected CTL immunotherapy for the treatment of B-cell non-Hodgkin's lymphomas. Am J Clin Exp Immunol. 2017; 6(3):27-42.

13. Fujita J, Mestre JR, Zeldis JB, Subbaramaiah K, Dannenberg AJ. Thalidomide and its analogues inhibit lipopolysaccharide-mediated induction of cyclooxygenase-2. Clin Cancer Res. 2001;7(11):3349-3355.

14. Böll B, Borchmann P, Topp MS, et al. Lenalidomide in patients with refractory or multiple relapsed Hodgkin lymphoma. $\mathrm{Br} \mathrm{J} \mathrm{Haematol.}$ 2010;148(3):480-482.

15. Fehniger TA, Larson S, Trinkaus K. A Phase 2 multicenter study of continuous dose lenalidomide in relapsed or refractory classical Hodgkin lymphoma. Blood. 2012;120(21):1623.

16. Mandac I, Kolonic SO. Lenalidomide induced good clinical response in a patient with multiple relapsed and refractory Hodgkin's lymphoma. J Hematol Oncol. 2010;3:20.

17. Marschner N, Zaiss M. Long-term disease stabilization in a patient with castration-resistant metastatic prostate cancer by the addition of lenalidomide to low-dose dexamethasone and celecoxib. Onkologie. 2012;35(5):279-282.

18. Tsai YC, Wu CT, Hong RL. Response of refractory osteosarcoma to thalidomide and celecoxib. Lancet Oncol. 2005;6(12):997-999.

19. Prince HM, Mileshkin L, Roberts A, et al. A multicenter Phase II trial of thalidomide and celecoxib for patients with relapsed and refractory multiple myeloma. Clin Cancer Res. 2005;11(15):5504-5514.

20. García-Recio M, Martinez-Serra J, Bento L, et al. Lenalidomide, celecoxib, and azacitidine therapy for blastic plasmocytoid dendritic cell neoplasm: a case report. Onco Targets Ther. 2016;9:5507-5511. 
OncoTargets and Therapy

\section{Publish your work in this journal}

OncoTargets and Therapy is an international, peer-reviewed, open access journal focusing on the pathological basis of all cancers, potential targets for therapy and treatment protocols employed to improve the management of cancer patients. The journal also focuses on the impact of management programs and new therapeutic agents and protocols on The manuscript management system is completely online and includes a very quick and fair peer-review system, which is all easy to use. Visit http://www.dovepress.com/testimonials.php to read real quotes from published authors.

Submit your manuscript here: http://www.dovepress.com/oncotargets-and-therapy-journal 